\title{
Biomarkers in Parkinson's disease
}

\section{Grace Crotty}

Department of Medicine, UCC

\section{Introduction}

Do you know anyone who has been diagnosed with Parkinson's disease? What is Parkinson's disease? In the Department of Medicine in UCC I am carrying out research on this condition, and I'm working with people who have Parkinson's disease (PD). I hope to increase our understanding of the disease mechanisms underlying PD along with identifying potential biomarkers for PD. What are biomarkers? Why do we need a biomarker? Keep on reading to discover more!

\section{What is Parkinson's disease?}

It is the second most common age-related neurodegenerative condition after Alzheimer's disease. It affects $1 \%$ of people over 65 years and $4 \%$ over 80 years. 30 million people are currently affected worldwide. It was first described by Dr. James Parkinson in 1817 in his book "An Essay on the shaking palsy". Parkinson's disease occurs when there is not enough of a chemical called dopamine being produced by your brain. People with PD classically present to their doctor with one of its cardinal motor features of resting tremor, slowness of movement or trouble with walking. However, other non-motor symptoms may also be present in addition to these motor features. These non-motor symptoms include difficulties with sleep, memory and mood along with dysfunction of the gastrointestinal, cardiovascular and autonomic nervous system. Interestingly, these non-motor symptoms, although quite nonspecific, may affect the patient before the onset of motor symptoms.

\section{How do you diagnose Parkinson's disease?}

Parkinson's disease is currently diagnosed by a doctor following the development of motor symptoms consistent with PD. We have no blood test or brain imaging technique that can diagnose PD before a significant amount of brain degeneration has already taken place. Therefore there can be a misdiagnosis of PD in 10-30\% of cases, particularly in the earliest stages of the condition. In some cases it can be difficult to know for sure that a person with a tremor definitely has PD rather than another neurological condition. We can only be $100 \%$ sure of the diagnosis of PD on post-mortem brain biopsy where we can see 
loss of the nerve cells producing a chemical messenger called dopamine, along with the presence of "Lewy bodies" which are structures containing deposits of a protein called alpha-synuclein.

Of note, PD can be present in a patient up to decades before the motor symptoms start. However, by the time a patient with PD develops these symptoms, more than $80 \%$ of the dopamine in their brain is gone. We currently have not identified any marker in the blood or spinal fluid to help us with diagnosing PD at a very early stage prior to the onset of symptoms. Research is ongoing to find a biomarker to do this.

\section{What is a biomarker?}

A biomarker is a parameter or an indicator of a particular disease state or a particular state of an organism. Biomarkers can be found anywhere in the body. They can be broadly classified into molecular biomarkers or imaging biomarkers. Whatever form the biomarker takes it should be simple, validated, inexpensive, as well as highly sensitive and specific for its particular condition.

\section{Why do we need a biomarker?}

Biomarkers are very important and useful for a variety of reasons. They can help with the early diagnosis of a condition, along with confirmation of diagnosis, population screening, drug development, monitoring of disease progression and of patients' responses to treatment. For people with PD a biomarker would be particularly helpful for identifying PD at an earlier stage in the disease process before the destruction of the nerves that produce dopamine in the brain. It would also increase our understanding of the underlying mechanisms of the disease and aid the development of neuroprotective strategies that could slow down or stop the disease process.

\section{Biomarkers in PD}

Our research group in University College Cork, along with several other research centres around the world, is currently searching for potential biomarkers for PD. The Michael J Fox foundation (www.michaeljfox.org) has also provided copious amounts of information and funding for research on PD. Biomarkers that have been studied can be subdivided into those that are preclinical, premotor, biochemical, genetic and imaging (Figure 1). 


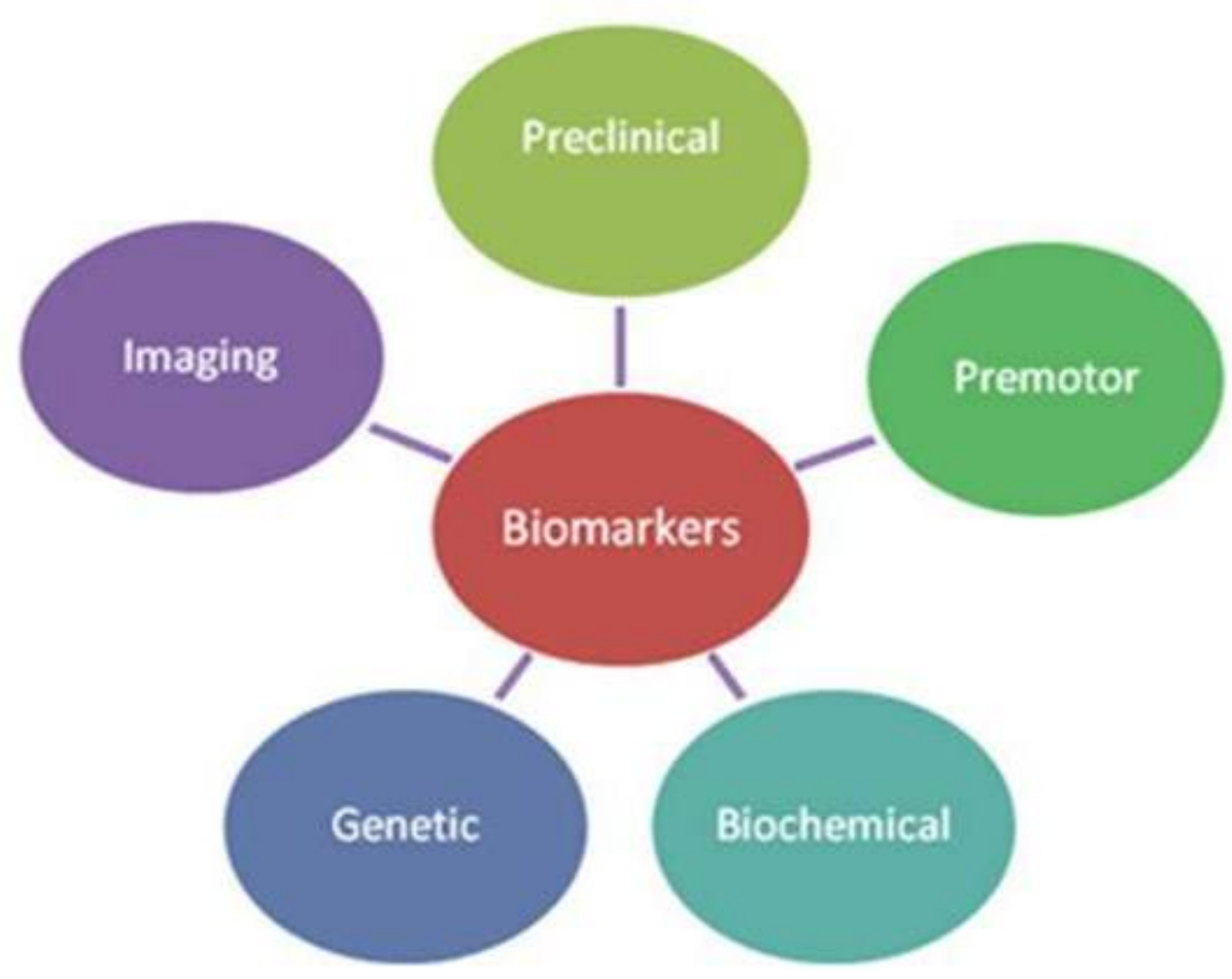

Figure 1: Biomarkers in PD. Image: Grace Crotty

\section{Preclinical biomarkers}

It is now well-recognised that people can have PD for years before they show any major symptoms. This is called the preclinical phase as they have not yet developed symptoms commonly associated with PD. Preclinical biomarkers look at risk factors that may increase a person's risk of later developing PD. Previous research has looked at patients with a positive family history for PD or abnormal brain imaging as risk factors for later development of PD.

\section{Premotor biomarkers}

These biomarkers look at symptoms other than the classical motor features that can be present pre-diagnosis with PD. Many potential biomarkers that have been identified including loss of smell, depression, restless legs and abnormal sleep activity. Unfortunately, a lot of these non-motor symptoms are quite common and can coexist in the elderly or with other diseases and, as such, are not specific to PD. 


\section{Biochemical biomarkers}

Examination of various bodily fluids and tissues samples has been carried out to investigate differences between people with PD and those without. In these samples you can analyse and measure genes, proteins, fats and other small molecules. Alterations in the levels of alpha-synuclein, anti-oxidative stress markers, urate and a protein called DJ-1 have been considered as potential biomarkers.

\section{Genetic biomarkers}

Approximately 10 percent of cases of PD are genetic. Using gene sequencing technology multiple new gene mutations have been identified. They include mutations in LRRK2, SNCA and parkin which are genes that are all felt to play roles in PD. Ongoing research has also investigated alterations in the genes responsible for the protein alpha-synuclein which is found in the PD brain on post-mortem biopsy.

\section{Imaging biomarkers}

Multiple imaging techniques have been looked at in order to identify unique changes in PD. Ultrasound imaging using sound waves, along with SPECT and PET imaging which use radioactive substances to trace activity in the nerve cells that produce dopamine, have noted changes in the brain consistent with PD. Magnetic Resonance Imaging (MRI) has also demonstrated increased iron content in the brain of patients with PD.

\section{Biomarkers in UCC}

In the Department of Medicine, I am currently carrying out three different studies on biomarkers in PD. I am recruiting both people with PD and control subjects. I'm recruiting all ages because although PD more commonly affects the elderly, it can occur in people at any age. All of my studies are looking for differences between people with PD and people without it. By identifying these differences it may help me to understand more about the underlying disease mechanisms of PD and hopefully find potential biomarkers.

\section{Non-motor symptoms}

My first study is a questionnaire study which compares the presence of non-motor symptoms in people with PD with those of controls. The questionnaires include questions on gastrointestinal symptoms, sleep disturbances, pain and impulse control disorders. From 
research, alterations in sleep and smell along with constipation occur frequently in people with PD.

\section{Autonomic symptoms and pain}

My second study is looking at autonomic symptoms and pain perception in people with PD compared to controls. Autonomic symptoms include problems with the way in which our nerves control our heart rate, breathing, temperature regulation and sweating. Participants fill out questionnaires on pain, autonomic symptoms and mood disturbance. They then undergo nerve conduction testing and thermal threshold measurements in order to assess the function of the nerves in their legs which are involved with movement, general sensation and temperature sensation. From research, it has been shown that the protein alpha-synuclein can also be found in the nerves of people with PD and may consequently affect nerve function.

\section{Cerebrospinal fluid biomarkers}

In my final study I'm measuring the levels of particular proteins in both the blood and spinal fluid in people with PD and controls. By doing this study I may detect a difference in the level of proteins between the two groups which can help with the earlier diagnosis of PD. In order to attain samples all participants undergo a blood test along with a spinal tap. A spinal tap is similar to an epidural that women would sometimes receive when giving birth. Both procedures are well-tolerated with minimal complications. It takes about thirty minutes in total to carry out these procedures on a general hospital ward. Following these tests, all participants will remain in hospital for a few hours with discharge home later that day.

\section{Conclusion}

It is an exciting time to be involved with research in Neuroscience, in particular Parkinson's disease. Investigative studies are being carried out throughout the world including in Cork, Ireland. PD is an age-related neurodegenerative disease and the number of people affected with PD will continue to increase as people live to older ages. Due to the ageing phenomenon, it is predicted that the proportion of the Irish population over 65 years of age will increase from $11.2 \%$ in 2002 to $27.6 \%$ in 2050 . It is therefore crucially important that we identify biomarkers and neuroprotective strategies to help prevent, cure or slow down the disease process in PD. In the Department of Medicine in UCC, I hope to identify differences between people with PD and those without. By doing so, we will learn more 
about the underlying disease mechanisms in PD and contribute towards the development of biomarkers in PD.

Thanks to my supervisors Dr. Sean O'Sullivan, Dr. Ger O'Keeffe and Dr. Aideen Sullivan without whom none of this work would be possible. I would also like to thank the Department of Medicine for the funding provided through the Professor Denis O'Sullivan fellowship grant. Last but not least I would like to thank the patients and their families without their generous participation in our research, none of this work would be possible. Figures were composed by the author who has permission for all images used. 